\title{
Pompéi. M. Tullius et le temple de Fortune
}

\section{Auguste}

Campagnes de fouilles et d'études 2011

\section{William Van Andringa}

École française de Rome et Université de Lille 3 (UMR 8164 du CNRS HALMA-IPEL) avec la collaboration du Musée Oiasso d'Irun et de l'Association Arkeolan et de la Soprintendenza speciale per i Beni Archeologici di Napoli e Pompei (éd.)

\section{(2) OpenEdition Journals}

Édition électronique

URL : http://journals.openedition.org/cefr/355

DOI : 10.4000/cefr.355

ISSN : 2282-5703

Éditeur

École française de Rome

Référence électronique

William Van Andringa, "Pompéi. M. Tullius et le temple de Fortune Auguste », Chronique des activités archéologiques de l'École française de Rome [En ligne], Les cités vésuviennes, mis en ligne le 19 décembre 2012, consulté le 11 septembre 2019. URL : http://journals.openedition.org/cefr/355; DOI : 10.4000/cefr.355

Ce document a été généré automatiquement le 11 septembre 2019.

(c) École française de Rome 


\title{
Pompéi. M. Tullius et le temple de Fortune Auguste
}

\author{
Campagnes de fouilles et d'études 2011
}

\section{William Van Andringa}

École française de Rome et Université de Lille 3 (UMR 8164 du CNRS HALMA-IPEL) avec la collaboration du Musée Oiasso d'Irun et de l'Association Arkeolan et de la Soprintendenza speciale per i Beni Archeologici di Napoli e Pompei (éd.)

\section{NOTE DE L'ÉDITEUR}

En 2011, l'équipe était composée de William Van Andringa, dir. (HALMA-IPEL, Université de Lille 3), Javier Arce (Université de Lille 3, Halma-Ipel), Jean-François Bernard (École française de Rome), Carole Chevalier, Sylvie Coubray (Inrap), Arnaud Coutelas (ArkeMine), Thomas Creissen (Université de Tours), Franck Decanter (Inrap), Xavier Deru (Université de Lille 3, Halma-Ipel), Vincent Drost (Université de Paris 1), Ingrid Dunyach (Université de Perpignan), Djamila Fellague (Université de Grenoble 2), Pauline Gaubert, Johannes Laiho (Helsinki), Tuija Lind (Helsinki), Christophe Loiseau (Université du Maine), Véronique Matterne (CNRS), Maria Jose Noain (Musée Oiasso d'Irun), Tarek Oueslati (CNRS, Halma-Ipel), Emmanuelle Rosso (Université d'Aix-Marseille), Eloisa Uribarri (Arkeolan), Maria Mercedes Urteaga (Musée Oiasso d'Irun), Anne-Sophie Vigot (EVEHA), Elena Zangitu (Arkeolan) avec la collaboration de Gilberte Chauffot (Université de Tours), Betta Interdonato et Violaine Monmarché (Université de Tours).

Cette présentation, tirée des rapports de fouilles déposés à la Soprintendenza Archeologica di Pompei, est le fruit d'un travail collectif auquel ont contribué l'ensemble des collaborateurs cités ci-dessus (cf. Van Andringa 2011). Il nous est agréable de remercier T. E. Cinquantaquattro, Surintendante et A. Varone, directeur de Pompéi, pour leur soutien à cette opération.

1 Comment étudier un lieu de culte de l'époque romaine? De quelle façon l'archéologie amène-t-elle à réévaluer notre connaissance des cultes antiques et de leur organisation ? Comment articuler l'étude architecturale ou archéologique d'un édifice religieux avec un 
discours historique sur le polythéisme qui vise généralement à restituer les modalités d'un langage extrêmement varié et complexe instauré entre les communautés et leurs dieux? Qu'apporte un examen stratigraphique rigoureux de l'environnement urbain d'un temple à la compréhension du lieu de culte? Ces questions sont fondamentales et amènent à poser les bonnes questions lorsqu'on étudie un lieu de culte. Pour faire court, on ne peut pas fouiller un sanctuaire comme on fouille n'importe quel habitat comme on ne peut pas étudier le polythéisme antique sans tenir compte des données archéologiques. La question est alors de savoir de quelles données archéologiques on dispose et de ce que l'on peut en faire.

2 Concernant le temple de Fortune Auguste à Pompéi, l'enquête a jusqu'ici privilégié une approche pluridisciplinaire de l'ensemble des données disponibles, architecturales et archéologiques, l'objectif étant de collecter le maximum d'informations sur le temple et les annexes, mais également sur son environnement urbain auquel il est nécessairement lié. C'est donc dans le cadre de cette collecte générale de l'information que nous devons désormais poser la question de l'utilisation des données qui intéressent un grand nombre de sujets, depuis l'évolution du quartier fouillé jusqu'à la construction du temple et ses aménagements successifs.

3 Le questionnement appliqué aux vestiges étudiés concerne alors trois domaines :

1. L'histoire religieuse, autrement dit l'histoire d'un culte public de Pompéi mis en place à l'époque augustéenne dans le contexte particulier de l'affirmation du Principat augustéen, à l'initiative d'un personnage bien connu de Pompéi, M. Tullius.

2. L'architecture d'un édifice monumental et l'organisation d'un chantier de construction antique, en notant que les publications de sanctuaires antiques ont pendant longtemps privilégié le domaine de l'architecture (il y a une raison à cela qui tient à la conception monumentale des temples romains construits en dur avec des sols de béton qui livrent souvent peu de témoignages sur la liturgie et le culte).

3. L'évolution d'un quartier urbain entre sa genèse à l'époque archaïque et la période impériale qui voit l'installation du lieu de culte. Se pose en particulier la question du choix du site pour l'implantation d'un temple en milieu urbain.

4 Ces trois directions d'enquête permettent de développer des thématiques propres qu'il est nécessaire de décloisonner de manière à restituer non pas seulement plusieurs sujets d'étude sur le culte de Fortune, la construction ou l'évolution de l'urbanisme, mais un mémoire d'histoire sur la fabrique urbaine d'un quartier, depuis les premières traces anthropiques (au $\mathrm{VI}^{\mathrm{e}} / \mathrm{V}^{\mathrm{e}}$ siècle av. J.-C.) jusqu'à la disparition du temple en 79 et sa redécouverte au XIX siècle, avec un moment fort, privilégié, qui voit l'installation d'un lieu de culte dédié à Fortune Auguste et l'inscription d'un culte public nouveau dans le calendrier de la colonie. Une telle approche a un avantage indiscutable, elle force à fondre la méthode archéologique dans une analyse historique globale.

5 L'étude du temple de Fortune Auguste et de ses annexes, entreprise en 2008, s'est poursuivie en avril/mai 2011, faisant intervenir une équipe de 24 personnes. La surface du podium a été fouillée sur la totalité de l'emprise du pronaos (secteur 6a) ainsi que sur deux quarts de la cella (secteur $6 \mathrm{~b}$ sud-est et nord-ouest). Le sondage installé dans la cella en 1910 a été ré-ouvert et continué jusqu'au soubassement du temple (secteur 6c). Un sondage installé à l'extrémité du couloir passant derrière le temple a permis de compléter les observations sur la structure du podium (secteur 13). L'étude de deux sondages des annexes ( $5 a$ et 7) implantés en 2010 a été achevée. Un sondage ouvert dans l'arrièreboutique de la caupona VII, 4, 4 avait pour but de compléter la longue coupe nord-sud et 
de travailler sur l'organisation et l'évolution du quartier dans lequel fut construit le temple de Fortune. Ce travail a été complété par des relevés architecturaux du temple, des annexes et des boutiques mitoyennes ainsi que par l'analyse des blocs d'ornement, des maçonneries et des techniques du bâti. Parallèlement au travail de terrain, le mobilier a été conditionné et étudié, les sédiments tamisés pour l'examen des restes carpologiques, anthracologiques et zoologiques. Une session d'étude au musée de Naples avait enfin pour objectif de recenser et d'étudier une partie du mobilier provenant du temple et découvert en 1824 (chapiteaux de pilastre, inscriptions, statues).

6 Les observations effectuées permettent de nourrir la problématique concernant l'évolution du quartier avant l'implantation du temple ainsi que les phases de chantier du lieu de culte (fig. 1).

Fig. 1 - Pompéi. Fouille du quart sud-est de la cella en cours, mise au jour des lambeaux de mortier d'attache des plaques du sol.

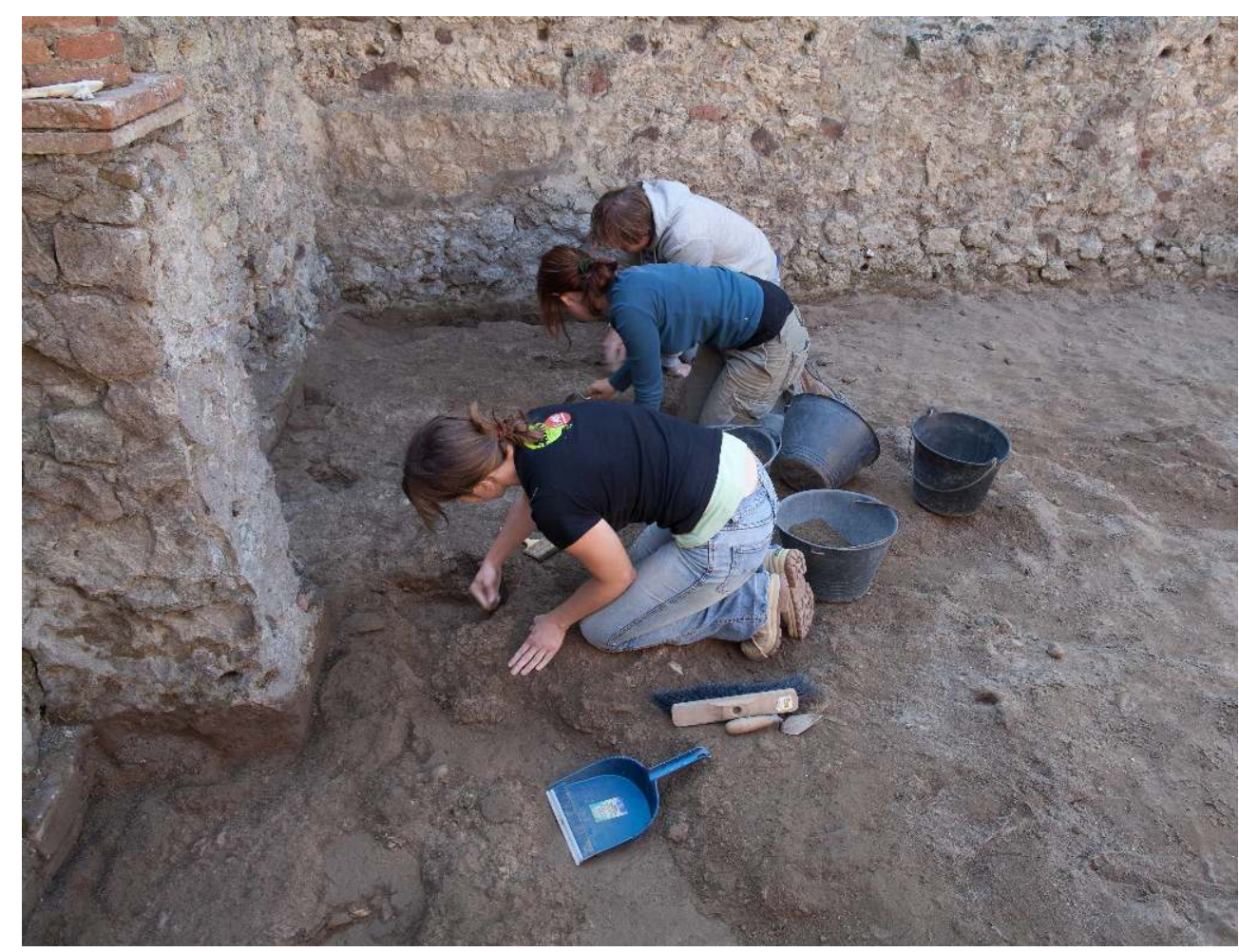

Cliché J. Laiho.

7 Sachant bien entendu que la lecture proposée ici relève de l'hypothèse de travail, en attendant l'étude complète du matériel céramique et la synthèse analytique des sondages effectués. Le sol naturel a été atteint à plusieurs endroits, notamment dans un sondage implanté le long du podium, livrant des paléosols dont le plus ancien reconnu est attribué par Marc Robinson à l'éruption de Mercato (7070 / 6770 av. J.-C.). Comme ailleurs dans la ville, l'occupation archaïque $\left(\mathrm{VI}^{\mathrm{e}}-\mathrm{V}^{\mathrm{e}}\right.$ siècle av. J.-C.) est installée sur le paléosol créé par la sixième éruption antéplinienne du Vésuve. Il est notable que les niveaux les plus anciens révélés autour du temple ne permettent pas pour l'instant de caractériser une occupation urbaine digne de ce nom avant le $\mathrm{III}^{\mathrm{e}}$ siècle av. J.-C. Comme dans l'insula voisine de la maison du Faune (VI, 12), ce siècle marque l'apparition des fondations de mur en grand appareil en calcaire de Sarno, l'occupation complète du quartier se poursuivant au IIe et 
Ier siècle avec une certaine frénésie comme l'indiquent les nombreuses phases de réaménagement mises en évidence dans la cour de la maison qui précède le temple (sondage 7). Nous sommes alors au cœur d'une ville en constante évolution et d'une vie urbaine très active.

Dans les vingt années qui précèdent le changement d'ère, $M$. Tullius prit l'initiative de faire bâtir un temple à Fortune Auguste sur la propriété qu'il possédait au carrefour des rues de la Fortune et du Forum. Dans un premier temps, il a fallu neutraliser et vider de ses habitants l'ensemble de la parcelle délimitée à l'est par le mur périmétral MR0031 de la casa delle Forme di Creta (VII, 4, 62), au sud, par le mur MR0033 dont on sait qu'il fut bâti à l'époque médio-républicaine pour servir de limite parcellaire jusqu'à l'éruption, à l'ouest et au nord par les deux rues perpendiculaires du carrefour (fig. 2).

Fig. 2- Pompéi. Plan du carrefour avant la construction du temple.

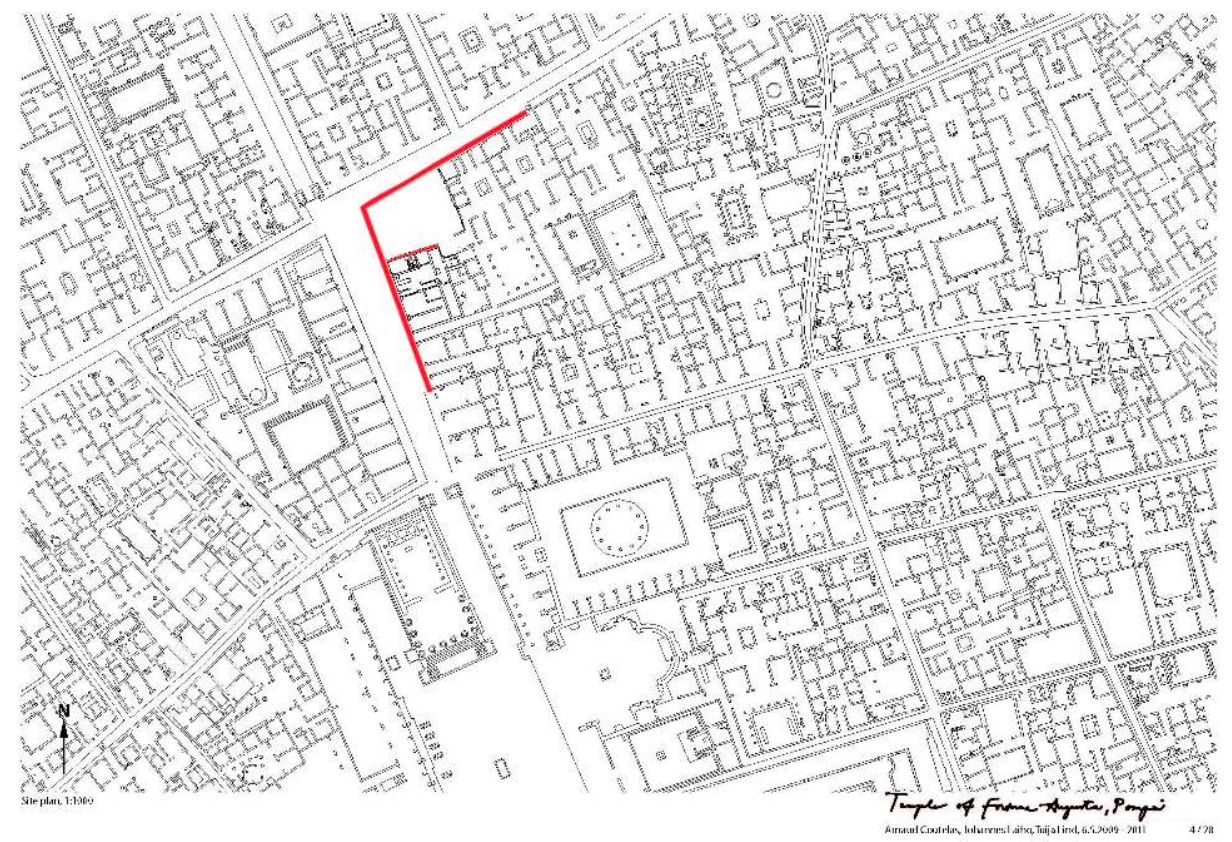

Les limites du chantier sont données au nord et à l'ouest par les rues de Fortune et du forum, au sud par le mur MR0033 (en rouge), à l'est par le mur périmétral de la casa delle Forme di Creta

Dessin J. Laiho et T. Lind.

9 Les espaces domestiques antérieurs appartenant à des boutiques et à une maison ont été arasés. On peut d'ailleurs noter que les laraires de la boutique attenante à la casa delle Forme di Creta ont été condamnés lors de l'opération. Les sols situés sur la partie sud de la parcelle, en dehors de l'emprise du podium, ont été réutilisés, un sol en ciment blanc servant d'appui à la structure en bois des machines de levage des blocs qui ont laissé l'empreinte des trous de poteaux et un sol en béton de tuileau orné de mosaïques repris pour servir de pavement à la salle de banquet des ministres de Fortune.

10 L'emprise du podium, élargie d'un espace nécessaire à la pose des coffrages de fondation, a ensuite été décaissée et nivelée jusqu'au terrain naturel (fig. 3). 
Fig. 3- Pompéi. Plan du carrefour après la construction du temple.

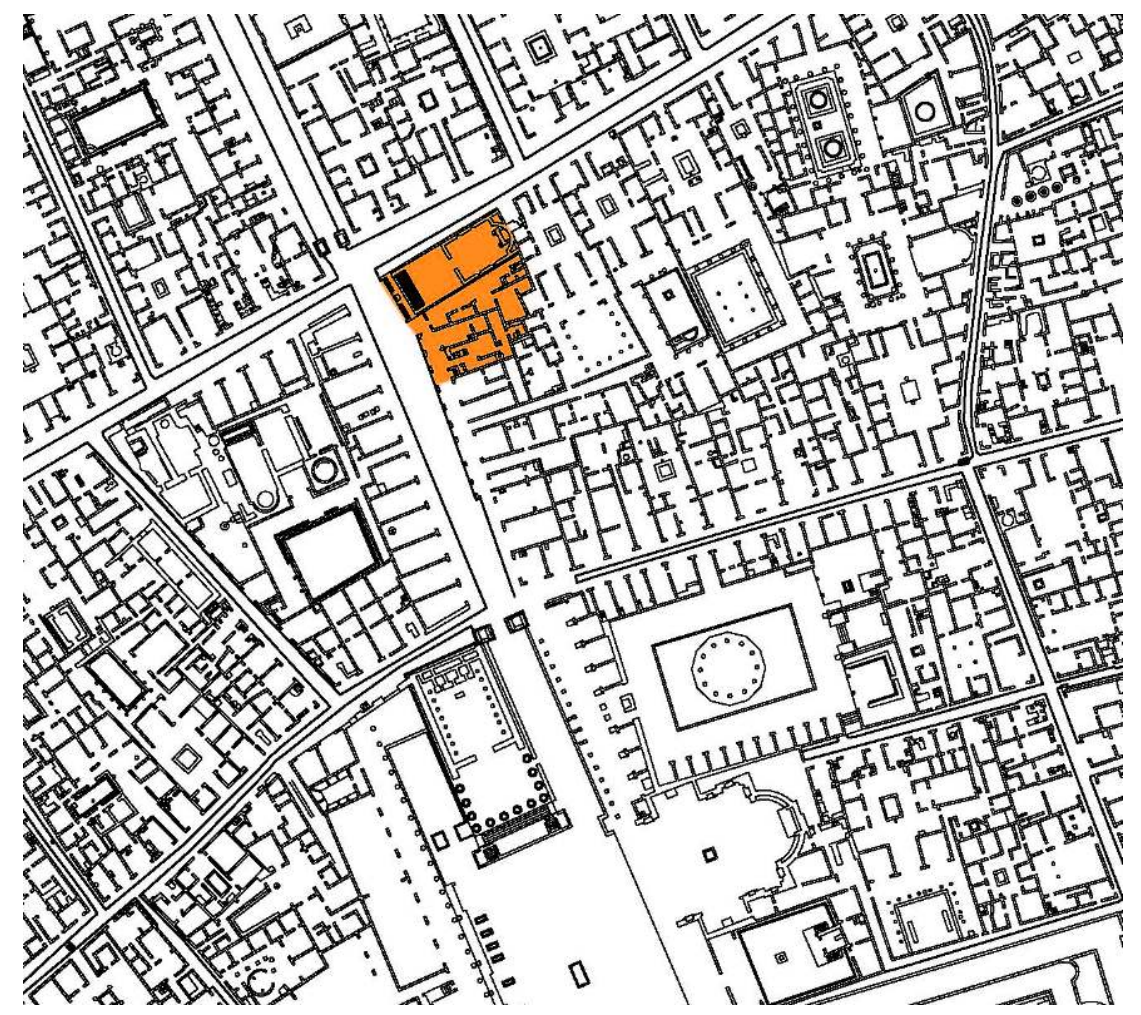

On remarque que l'escalier et la plate-forme de l'autel ont été construits sur la rue, provoquant un «redressement » vers l'ouest de la rue montant du forum

Dessin J. Laiho et T. Lind.

11 La tranchée de terrassement a certainement empiété sur les trottoirs à l'ouest et au nord, venant buter à l'est contre le mur de la maison mitoyenne, libérant par la même occasion des mètres cubes de remblais et de matériaux de destruction déposés à proximité (dans la rue ?). C'est à ce moment-là que furent tracées au sol les limites du podium. On peut alors se demander si une cérémonie d'inauguration n'a pas eu lieu, dirigée par M. Tullius en personne puisque celui-ci était augure et donc parfaitement qualifié pour « libérer » et «définir » le lieu. Si l'on accepte cette hypothèse, les limites augurales données au templum de Fortuna ont pu correspondre à la plate-forme du temple, à l'emprise de l'aedes. L'autre cérémonie que l'on attend à ce stade de l'entreprise est celle de la consecratio. Il s'agissait de préparer rituellement l'espace sur lequel allait être érigé le temple. Faut-il imaginer, sur l'exemple de la consécration du nouveau capitole de Rome (Tac. Hist. 4, 53), des piquets et des cordages, la pose des premiers pierres, quelques rites, le jet de monnaies par exemple, tout cela avec la présence du duumvir assisté du pontife, peut-être M. Lucretius Decidianus Rufus connu à la même époque par une base érigée sur le forum? Toutefois, aucune trace de telles cérémonies n'a pu être mis en évidence dans les différents sondages, sachant d'une part que l'observation est gênée par le présence de gaines techniques modernes installées en tranchée le long du podium et d'autre part que le soubassement du podium n'a pu être sondé que très partiellement. En effet, les autorités du site ont, en 1910, pour des raisons qui nous échappent, fait un sondage dans le nucleus en béton du podium pour découvrir un espace voûté souterrain. Après mise en sécurité au moyen d'échafaudages, le sondage a été repris et élargi à l'espace voûté du 
podium pour atteindre le terrain naturel ayant servi d'assise au temple. Ce qui a permis de préciser les techniques de construction employées (fig. 4).

Fig. 4 - Pompéi. Vue de l'un des caissons de la structure du podium du temple après enlèvement des échafaudages destinés à la sécurisation du sondage.

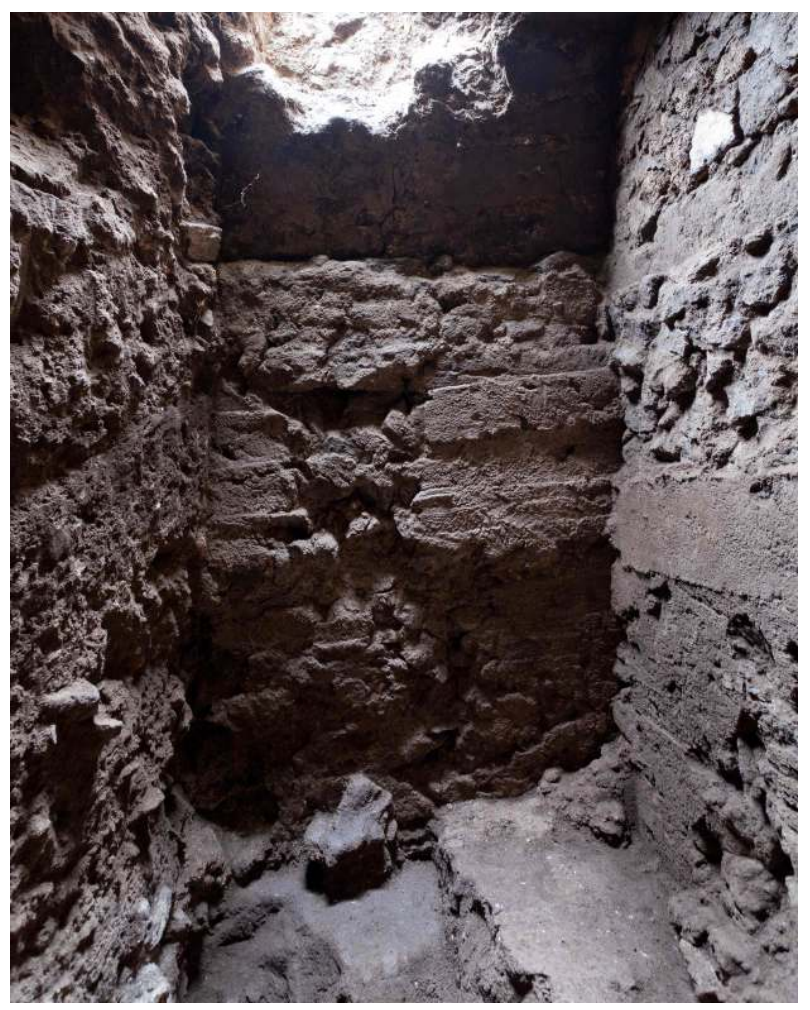

On distingue nettement les traces du coffrage ainsi que les remblais (à gauche) sur lesquels fut construite la voûte.

Cliché J. Laiho.

Une fois le nivellement effectué jusqu'au terrain naturel, les fondations du mur périmétral du podium ont été coulées dans des tranchées creusées dans le terrain naturel. Les fondations étant posées, des caissons ont été constitués en élévation à partir de murs transversaux bâtis en coffrage. Des remblais provenant des terrassements et de la destruction des bâtiments antérieurs ont été apportés pour combler progressivement l'intérieur des coffrages jusqu'à la constitution d'une assise de réglage sur laquelle fut appuyé le boisage de soutien de la voûte. Les voûtes des caissons ont ensuite été construites directement sur les remblais avant que ne soit coulé le béton du nucleus. Une fois le podium monté, les travaux ont concerné les maçonneries de la cella bâties en opus incertum recouvert d'une fine couche d'enduit. Ce sont ces travaux sans aucun doute qui ont laissé une myriade de trous de piquet et de poteaux dans le nucleus en béton, des traces que l'on est bien en mal d'interpréter pour l'instant (fig. 5). 
Fig. 5 - Pompéi. Fouille du quart sud-est de la cella.

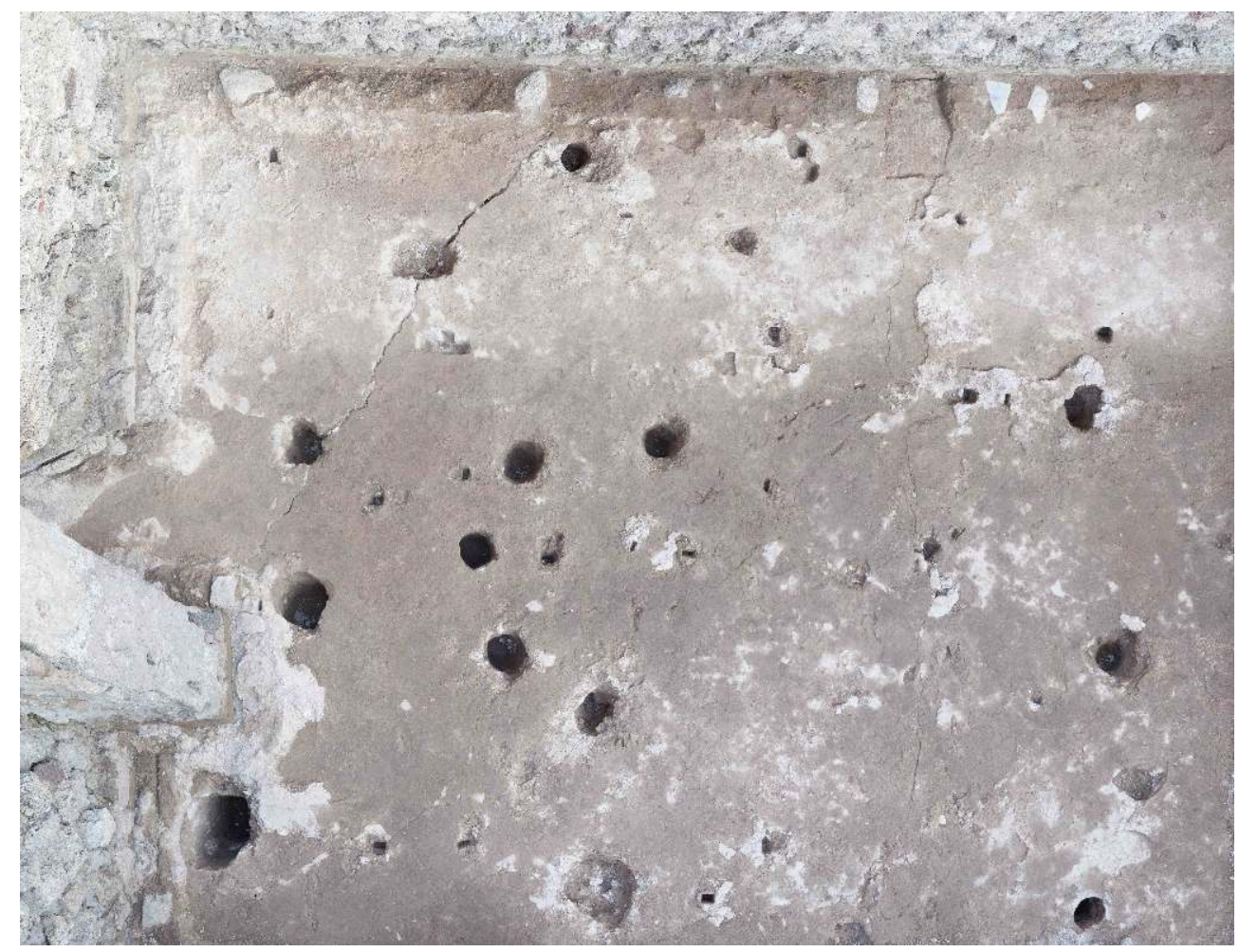

Vue du nucleus du podium percé de trous de poteaux et de piquets

Cliché J. Laiho.

13 Concernant l'emprise donnée au podium, on remarque que celle-ci respecte les limites de la propriété de M. Tullius, sauf peut-être dans l'angle nord-ouest (mais nous n'avons aucune idée à cet endroit de la largeur du trottoir antérieur au temple); autrement dit le parti pris lors de la construction du podium respecte à la lettre les termes du titulus du temple (CIL X, 820) qui indique que l'aedes (en l'occurrence ici la partie haute du temple, pronaos et cella) a été construite solo et pequnia sua, aux frais de Tullius et très exactement dans les limites de sa propriété. Ce n'est qu'une fois que la maçonnerie de l'aedes fut construite que l'on ajouta la rampe de l'escalier doublé par une plate-forme servant d'assise à l'autel sacrificiel. Les relevés ne laissent aucun doute: les concepteurs ont volontairement choisi de bâtir la plate-forme de l'autel directement et entièrement sur la rue et l'espace public, un acte à mettre en relation avec le statut public donné au culte installé : l'établissement de l'autel sur la rue donnait une emprise publique au culte institué (fig. 6). 
Fig. 6 - Pompéi. Maquette du secteur étudié montrant les élévations en place

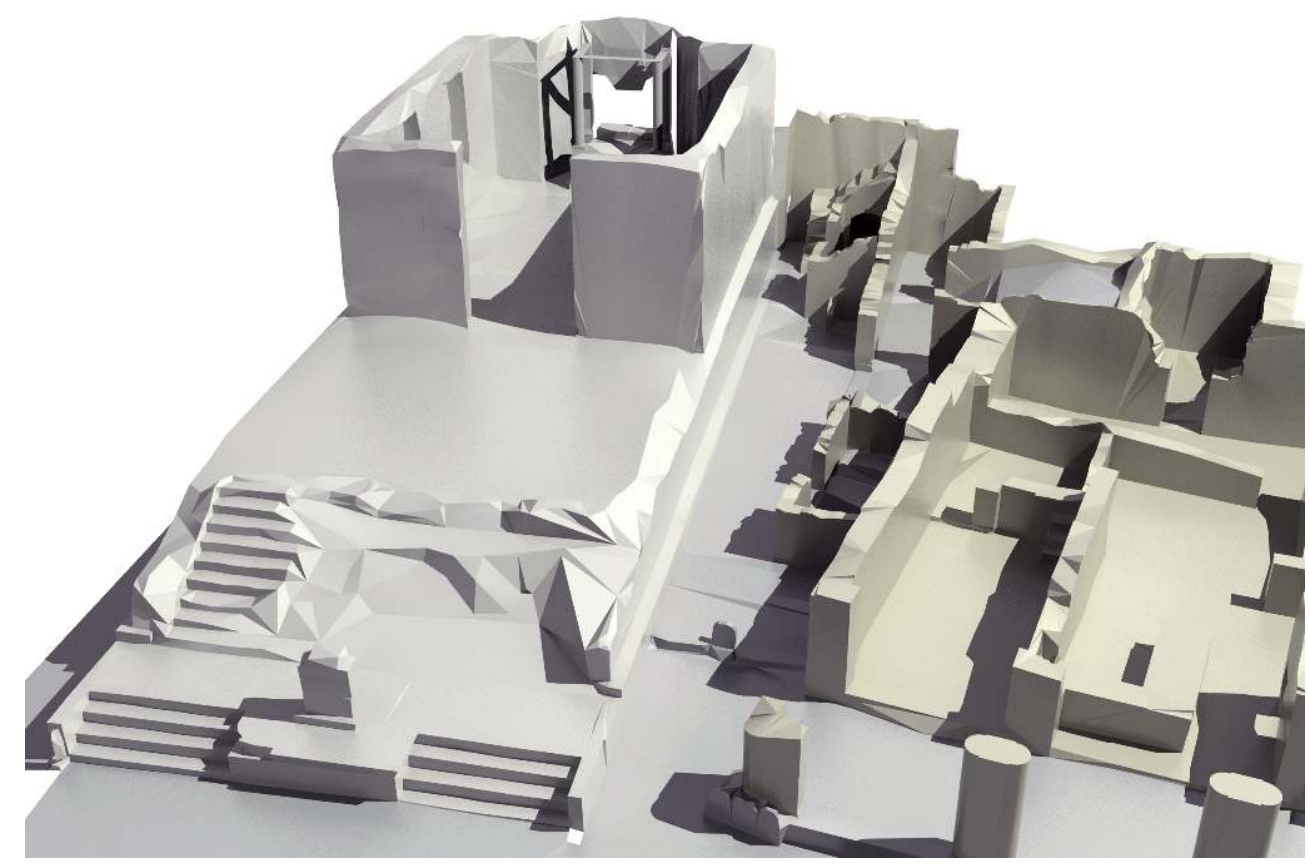

J. Laiho et T. Lind. des placages de marbre. Les blocs et plaques furent préparés sur place comme l'attestent les très nombreux éclats de marbre blanc retrouvés sur les niveaux de chantier, avant d'être montés grâce à des structures de levage qui ont laissé les empreintes au sol sous la forme de trous de poteaux relevés le long du côté sud du podium. Le dallage du pronaos et de la cella a été réalisé avec des plaques entièrement récupérées après l'éruption. À l'intérieur de la cella, les éclats de marbre découverts dans le niveau de mortier du sol montrent que la décoration utilisait des marbres colorés. Au même moment, on démontait les machines de levage installées en contrebas du podium; il s'agissait en effet de compléter les travaux par l'aménagement des annexes destinées aux ministres du culte. C'est précisément à ce moment-là, entre le démontage des machines de levage et l'aménagement des sols des annexes qu'intervint une cérémonie religieuse dont on a conservé quelques traces sous la forme de restes de fruits carbonisés enfouis dans le comblement d'un trou de poteau.

L'espace de la cella ayant déjà été consacré, rendu sacré par la cérémonie de la consecratio, les statues des dieux pouvaient y prendre place dans un ordonnancement précis qui donnait sens au culte institué 2 . La place principale, dans l'axe, face à l'autel fut donnée comme il se doit à la statue de culte qui fut dressée dans une abside monumentalisé dès la phase initiale de construction du temple. L'installation de Fortuna prenait toutefois place dans un contexte civique et politique particulier, ce qu'exprime la présence extraordinaire d'autres statues qui ont pris place également dans la cella, dans les quatre niches aménagées dans les deux murs latéraux. Les découvertes effectuées au XIX siècle permettent d'identifier au moins trois occupants qui partageaient la demeure de Fortuna. On trouvait Auguste reconnu grâce à une dédicace de statue le mentionnant comme parens patriae associé à un personnage masculin identifié à $M$. Tullius et à un drapé féminin en cours d'identification. Certes A. D. Nock a montré que parfois des personnages 
de premier plan pouvaient prendre place dans un temple, à proximité de la divinité titulaire, ce qui les assimilait à des sunnaoi theoi ${ }^{3}$. Mais cet honneur suprême était rare, réservé à des personnages d'exception comme ce médecin Antonius Musa qui ayant soigné Auguste a reçu l'honneur d'une statue placée à côté de la statue d'Esculape précise Suétone (Aug. 59), autrement dit à l'intérieur de la cella ${ }^{4}$. C'est également un honneur insigne que reçurent Auguste et $\mathrm{M}$. Tullius dont les statues furent installées à proximité de l'effigie de Fortuna. Certes, ces statues doivent être comprises comme des ornements ( ornamenta) associés au culte, manifestant la piété de l'évergète et de l'empereur, mais l'association n'en avait pas moins un sens religieux précieux qui unissait la déesse aux deux représentants de l'État romain et de la colonie de Pompéi.

Nous sommes en 3 ap. J.-C., voilà donc le temple fondé, les titulaires installés, un collège de ministres ordonné pour la gestion du culte et logé à proximité immédiate, même si hors de l'espace sacré proprement dit (CIL X, 824). Pouvait intervenir la cérémonie de dédicace qui entérinait définitivement le transfert de l'espace consacré et aménagé dans la propriété divine de la déesse Fortuna. Le magistrat de la cité, très certainement en présence de M. Tullius (si ce n'était lui-même!) et sous la dictée du pontife a énoncé publiquement le transfert du bien à la déesse (en tenant les montants de la porte de l' aedes), avant de définir le statut des biens affectés au sanctuaire et les règles rituelles. C'est sans doute à ce moment là que fut également publié le règlement du collège des ministres affectés à la gestion du culte par les autorités de la cité (CIL X, 825). La liste des quatre premiers ministres datée de 3 ap. J.-C. montre que la dédicace a pu avoir lieu à cette date qui correspond également à la proclamation publique du culte.

Le dossier du temple de Fortune Auguste à Pompéi, par les multiples développements qu'il autorise, montre ainsi que la fondation d'un temple ne se résume pas à une séquence rituelle, par ailleurs complexe, qui permettait la prise des auspices ou qui réglait le transfert du bien à la divinité choisie, mais qu'elle s'inscrit dans un contexte historique précis qui donnait un sens local au culte institué, ce qui est l'essence même du polythéisme. La déesse Fortuna avait colonisé tant d'endroits depuis l'instauration du culte par Servius Tullius à Rome, mais la Fortuna de M. Tullius installée à Pompéi n'était pas la Fortune de Servius Tullius installée à Rome ; Fortuna Augusta avait une coloration bien particulière donnée moins par les rites de l'inauguratio ou de la consecratio qui n'ont laissé aucune trace que par la personnalité du fondateur M. Tullius, proche d'Auguste et citoyen excellentissime de la colonie de Pompéi.

\section{BIBLIOGRAPHIE}

Arce 2011 = J. Arce, Fortuna Augusta, Auguste et Marcus Tullius, dans Van Andringa 2011, vol. 1.

Gavel 2011 = S. Gavel, Placing paniskoi herms within Augustan propaganda at Pompeii, dans JRA, 24, 2011, p. 191-214.

Nock 1972 = A. D. Nock, Synnaos theos, dans Essays on Religion and the Ancient World, Oxford, 1972, p. 202-251. 
Van Andringa 2011 = W. Van Andringa (dir.), Les lieux de culte de Pompéi : le temple de Fortune Auguste (VII, 4, 1-2) et les boutiques mitoyennes (VII, 4, 3-4), le fulgur conditum de la maison des Quatre Styles (I, 8, 17), Campagne 2011, rapport d'opération, Pompéi, 2 vol.

Van Andringa 2012 =W. Van Andringa, Statues in the Temples of Pompeii. Combinations of Gods, Local Definition of Cults and the Memory of the City, dans B. Dignas, B. Smith (éd.), Historical and Religious Memory in the Ancient Mediterranean World : Religion, Memory, History, Oxford, 2012, p. 83-115.

\section{NOTES}

1. L'accès à la plate-forme de l'autel était gardé par une barrière métallique dont sont conservées les attaches au sol. Cette balustrade a été arrachée lors des campagnes de récupération des matériaux postérieures à l'éruption. Aucun lien ne peut être fait en tout cas avec des hermès en bronze découverts (enfouis ?) dans un local de façade de la casa delle Forme di Creta (VII, 4, 60-61), contrairement à ce qu'indique un article récent, cf. Gavel 2011, p. 191-214.

2. Voir Van Andringa 2012, p. 83-115.

3. Voir Nock 1972, p. 202-251.

4. Voir Arce 2011.

\section{INDEX}

Mots-clés : lieu de culte, temple, architecture, espace urbain, histoire religieuse institutions École française de Rome, Université de Lille 3 (UMR 8164 du CNRS HALMA-IPEL), Musée Oiasso d'Irun, Soprintendenza speciale per i Beni Archeologici di Napoli e Pompei Index géographique : Pompéi

\section{AUTEURS}

\section{WILLIAM VAN ANDRINGA}

Université Lille 3 (UMR 8164 du CNRS HALMA-IPEL) - william.vanandringa[at]univ-lille3.fr 